\title{
Role model: Anita Thapar
}

\author{
Anne Gulland
}

\section{London}

When Anita Thapar began training as a child psychiatrist in the 1980s the myth that parents were to blame for their children's neurodevelopmental conditions persisted in some quarters. Some still believed the "refrigerator mother" hypothesis for autism-that a lack of maternal warmth and affection were responsible for a child's condition.

The idea that genetic factors were relevant to childhood psychiatric disorders was so unusual that when Thapar told her colleagues she was going to do her $\mathrm{PhD}$ in genetics some thought she was quitting psychiatry.

"At that time, genetics and psychiatry were poles apart," she says.

During her $\mathrm{PhD}$ she became interested in attention-deficit/hyperactivity disorder (ADHD).

"There's so much stigma around mental health and ADHD, and some people still think it's a made up condition. But there's lots of research, not just my work, which shows it does behave like a neurodevelopmental disorder. The brain is affected, it starts early on, and there is a close overlap with autism, intellectual disability, and learning and language problems." Thapar says.

"In Wales, specific child neurodevelopmental services are being set up-which I think is going to be great, given the strong clinical and biological overlap between ADHD and these other disorders. Although genes have been found to contribute to ADHD, like most common disorders it's a complex mix of environment and genetics," she says.

Thapar is excited that genetics and clinical medicine are coming together. "Parents in clinic say the research on ADHD has been helpful in reducing the stigma," she says.

ADHD, particularly its treatment with drugs, can be a controversial topic. Some commentators are uneasy with the idea of a genetic link to the condition because they worry that this will lead to purely pharmacological, rather than behavioural, treatment. However, this is a misunderstanding of genetics, says Thapar.

She says that National Institute for Health and Care Excellence guidance, which recommends a blend of psychological interventions, education programmes, and drugs for the more severely affected, is "sensible." The use of drugs has begun to increase but the other recommended interventions are not widely available, she says. "I hope that psychiatry doesn't become a prescribing only specialty. Drugs can be life saving for some. However, medicine is the art of blending a pharmaceutical agent with lifestyle measures," she says.
Thapar was born in South Wales and educated in Cardiff, where she also went to university and did the early part of her clinical training. She moved to west Wales with her husband, a GP trainee at the time, where she finished her clinical training. Working in district general hospitals in Carmarthen and Swansea may seem an inauspicious start to an academic career but Thapar says that it gave her an excellent clinical grounding.

After she completed her $\mathrm{PhD}$-which took a bit longer than she intended, as she had her first child during her research - she moved to Manchester for a senior lecturer post. This was a busy time both professionally and personally, and while she was pregnant with her second child she was appointed the first professor of child and adolescent psychiatry at Cardiff University.

"It started off as just me and a PhD student but now it's grown. The first thing I had to do was revamp the undergraduate teaching programme and then develop links with clinical colleagues. However, because I had been a trainee in Cardiff there was a lot of goodwill and support from my NHS colleagues," she says.

She arranged for specialist registrars to rotate through her academic group and has retained links with many now they are consultants.

"It's good to keep the links with the NHS strong-it's important to me that our research is not just done by people sitting in an office in the university and that clinical colleagues understand what academics do and are involved in research," she says.

Her two sons have now grown up and Thapar is in a new phase of her career-she's able to travel more and has begun new clinical and research work. After a chance conversation with a neonatal professor she is going to start research on the links between pre-term birth and autism and ADHD, and the interplay with genetics. She is also conducting new research on the natural history of ADHD and autism in people from 4 to 25 years old.

But an important part of her role is looking at the next generation of researchers and academics.

"I love mentoring researchers, trainee doctors, and medical students-it's one of the most rewarding activities I do. I have had a lot of help in terms of people opening doors for me, so I want to do the same for them," she says. 


\section{Nominated by Jude Harrison}

Harrison says, "Anita Thapar specialises in the neurobiology of neurodevelopmental disorders, particularly genetics. As a female, tenured,

clinical academic professor she represents a minority. In addition to her world leading research and clinical commitments, Anita takes time to mentor junior female clinical academics. She is a fantastic role model for women in clinical research."

Jude Harrison is a clinical academic training fellow, Cardiff University 